\title{
The many faces of early-type dwarf galaxies
}

\author{
T. Lisker, E. K. Grebel, B. Binggeli, M. Vodička, K. Glatt \\ and P. Westera
}

Astronomical Institute, Dept. of Physics and Astronomy, University of Basel, Switzerland

\begin{abstract}
We present a study of 413 early-type dwarf (dE) galaxies in the Virgo Cluster with SDSS multicolour imaging. Several dE subclasses show systematic differences in shape, clustering properties, and stellar content. We find different colour-magnitude relations for nucleated and non-nucleated $\mathrm{dEs}$, as well as for $\mathrm{dE}$ samples populating regions of different densities.
\end{abstract}

Keywords. Galaxies: dwarf, galaxies: structure, galaxies: stellar content

\section{Introduction}

How do early-type dwarf ( $\mathrm{dE}$ ) galaxies form? These small, low surface brightness objects are the most numerous galaxies in clusters, and are thus ideal probes to study the physical processes that govern galaxy formation and evolution in such environments. Their formation mechanisms are, however, still a matter of debate (see Jerjen \& Binggeli 2005, and references therein). Proposed scenarios include ram-pressure stripping (Gunn \& Gott 1972) of dwarf irregulars, as well as harassment (Moore et al. 1996) of infalling spirals. If the dEs carried the imprint of these processes in their structure and stellar content, they could serve as tracers of cluster assembly and evolution. Thus, the goal of our analysis of 413 Virgo cluster dEs with optical imaging data from the Sloan Digital Sky Survey (SDSS; Adelman-McCarthy et al. 2007) is to systematically study their structural and stellar characteristics, and relate these to their distribution within the cluster. After having established a subdivision scheme for dEs into subclasses with different shapes and distributions (Lisker et al. 2007; see Section 3), we can now proceed with analyzing their colours and stellar populations. Here, we present first results of this analysis.

\section{Data and sample}

The SDSS imaged almost all galaxies of the Virgo cluster catalog (VCC; Binggeli et al. 1985 ) in five optical bands, $u, g, r, i$, and $z$. We determined the sky flux distribution $\dagger$ across a given image using a thorough procedure, as described in detail in Lisker et al. (2007). The sky-subtracted images were then flux calibrated, with the required parameters provided by the SDSS, and corrected for Galactic extinction (Schlegel et al. 1998). We also correct for the reported SDSS zeropoint offsets in the $u$ and $z$ bands from the AB system (see http://www.sdss.org/dr5/algorithms/fluxcal.html).

Our working sample of Virgo cluster dEs contains 413 certain cluster members that were initially classified as early-type dwarfs in the VCC ("dE" or "dS0"), that are brighter than $m_{\mathrm{B}} \leqslant 18.0 \mathrm{mag}$, that passed our visual examination for possible dwarf irregulars,

$\dagger$ The SDSS photometric pipeline significantly overestimated the local sky flux around the Virgo dEs due to their large apparent sizes and low surface brightness outskirts. This affected its derivation of isophotal and Petrosian radii, its profile fits, and subsequently its calculation of total magnitudes, which can be wrong by up to 0.5 mag. Therefore, we do not use any of the photometric measurements provided directly by the SDSS. 
and for which a Petrosian radius could be derived. See Lisker et al. (2007) for details. Note that we include both "dwarf ellipticals" and "dS0s" of the VCC in our sample of early-type dwarfs, and use the common abbreviation "dE" for all of them, in order to avoid any preselection bias. Total galaxy magnitudes in the $r$ band are measured within an elliptical aperture of two "Petrosian semimajor axes", i.e., ellipses instead of circles were used in the calculation of the Petrosian radius (Petrosian 1976; Lotz et al. 2004). Colours are measured within the (elliptical) half-light aperture.

\section{Shapes and distributions}

In Lisker et al. (2007), we presented a quantitative analysis of the intrinsic shapes and spatial distributions of various dE subclasses: bright and faint nucleated and nonnucleated $\mathrm{dEs}(\mathrm{dE}(\mathrm{N}) \mathrm{s}$ and $\mathrm{dE}(\mathrm{nN}) \mathrm{s})$, dEs with disk features like spiral arms or bars (dE(di)s; Lisker et al. 2006a), and dEs with blue centers (dE(bc)s; Lisker et al. 2006b). The $\mathrm{dE}(\mathrm{bc}) \mathrm{s}, \mathrm{dE}(\mathrm{di}) \mathrm{s}$, and bright $\mathrm{dE}(\mathrm{nN}) \mathrm{s}$ are shaped like thick disks and show no central clustering, indicating that they are an unrelaxed population that probably formed from infalling progenitor galaxies. In contrast, the $\mathrm{dE}(\mathrm{N}) \mathrm{s}$ are a fairly relaxed population of spheroidal galaxies, though an oblate intrinsic shape is favored for them as well. The faint $\mathrm{dE}(\mathrm{nN}) \mathrm{s}$ appear to be somewhat intermediate: their shapes are similar to the $\mathrm{dE}(\mathrm{N}) \mathrm{s}$, but they form a largely unrelaxed population, as derived from their clustering properties. Taken together, these results define a morphology-density relation within the dE class.

\section{Colour analysis}

The colours of different $\mathrm{dE}$ subclasses cannot straightforwardly be compared with each other due to the colour-magnitude relation $(\mathrm{CMR})$ : their average colour values could be different only because of a different sampling in magnitude. For this reason, we analyze the $\mathrm{dE}$ colours through a comparison of various CMRs. Linear fits to the CMRs are done by means of a robust parameter estimation technique, namely a so-called M-estimate based on the mean absolute deviation (Press 2002).

Figure 1 shows the CMRs of the $\mathrm{dE}$ subclasses. In all colours $(u-r, g-i$, and $i-z)$, the CMR of the $\mathrm{dE}(\mathrm{nN}) \mathrm{s}$ is steeper than that of the $\mathrm{dE}(\mathrm{N}) \mathrm{s}$. Moreover, the $\mathrm{dE}(\mathrm{N}) \mathrm{s}$ show a smaller scatter about the CMR, which is not only due to the fact that they are, on average, brighter than the $\mathrm{dE}(\mathrm{nN}) \mathrm{s}$ and thus have smaller measurement errors. While the CMRs of $\mathrm{dE}(\mathrm{N}) \mathrm{s}$ and $\mathrm{dE}(\mathrm{nN}) \mathrm{s}$ intersect at fainter magnitudes, their slope is different, such that the brighter $\mathrm{dE}(\mathrm{nN}) \mathrm{s}$ are bluer than the brighter $\mathrm{dE}(\mathrm{N}) \mathrm{s}$ in all colours. Given that $u-r$ is mainly age-sensitive, while $i-z$ is metallicity-sensitive, the brighter $\mathrm{dE}(\mathrm{nN}) \mathrm{s}$ seem to be both younger and less metal-rich than the brighter $\mathrm{dE}(\mathrm{N}) \mathrm{s}$.

The CMR of the $\mathrm{dE}(\mathrm{di}) \mathrm{s}$ is more similar to that of the $\mathrm{dE}(\mathrm{N}) \mathrm{s}$ in all colours. However, note that it is defined by only three data points at the faint end. Likewise, the CMR of the $\mathrm{dE}(\mathrm{bc}) \mathrm{s}$ is only constrained by two data points at the faint end. Nevertheless, both the $\mathrm{dE}(\mathrm{di}) \mathrm{s}$ and $\mathrm{dE}(\mathrm{bc}) \mathrm{s}$ form a strikingly tight CMR in $i-z$, whereas they show a large scatter in $g-i$. This might indicate that each of these two dE subclasses covers a rather narrow range in metallicity.

Figure 2 shows the CMRs of the low- and high-density dE sample, using all dEs except the $\mathrm{dE}(\mathrm{bc}) \mathrm{s}$, and separating the samples at the median value of local projected galaxy number density (19.6 per square degree), calculated from a circular area enclosing the ten nearest neighbour galaxies. In all colours, the low-density CMR is slightly steeper than the high-density one, implying that the density distribution within the cluster had at least some effect on the star formation history of the dEs. 


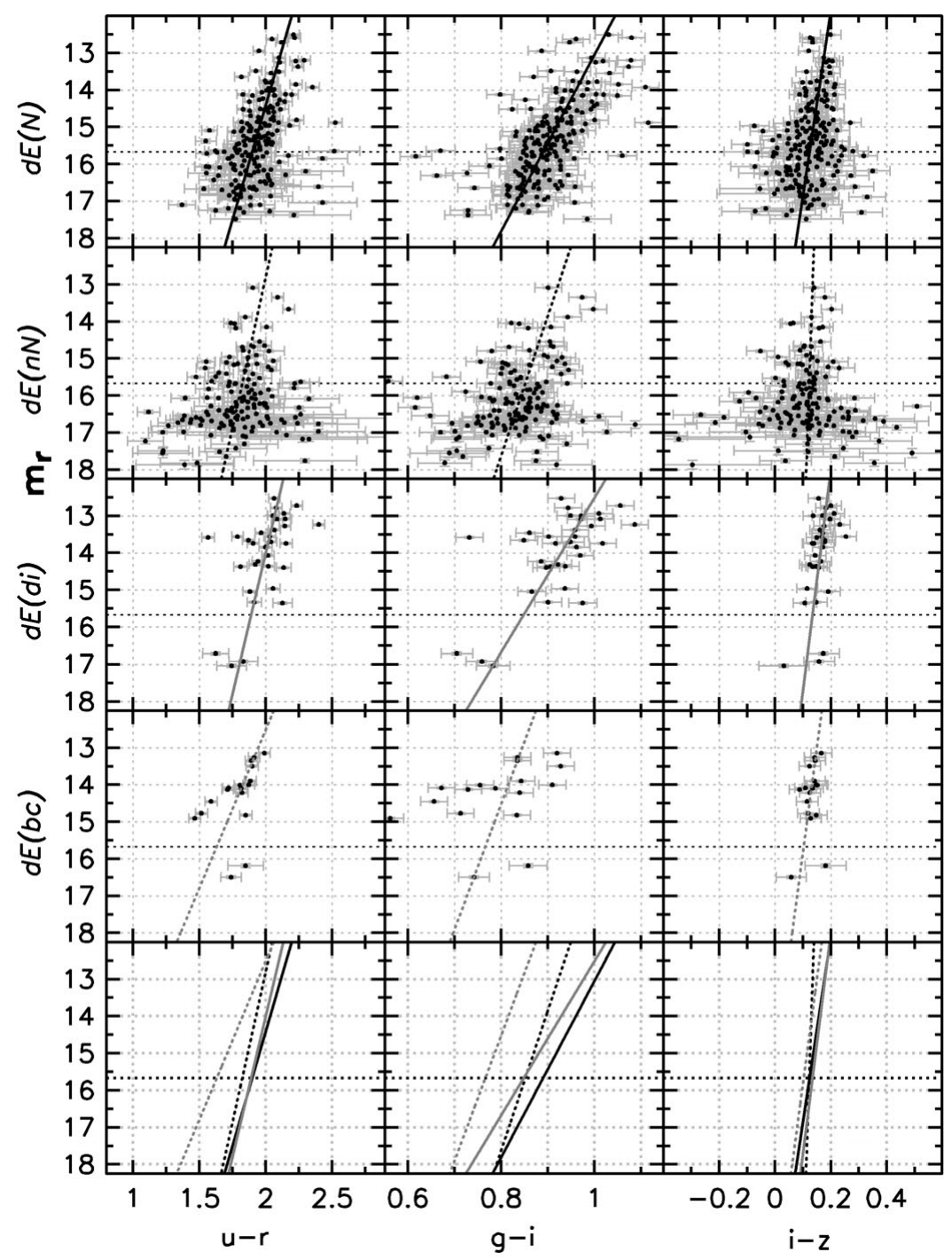

Figure 1. Colour-magnitude relations of dE subclasses. Shown are the relations of $r$ magnitude with $u-r$ (left), $g-i$ (middle), and $i-z$ (right) for the different $\mathrm{dE}$ subclasses (different rows, as labelled on the left-hand side). Colours are measured within the half-light aperture. Each linear fit is plotted again in the bottom row for comparison.

\section{Summary and Outlook}

We have presented first results of a colour analysis of 413 Virgo cluster dEs, for which we constructed colour-magnitude relations (CMRs) for different dE subclasses (see Lisker et al. 2007) and different local densities. In all colours $(u-r, g-i, i-z)$, we find differences between the CMRs of $\mathrm{dE}(\mathrm{N}) \mathrm{s}$ and $\mathrm{dE}(\mathrm{nN}) \mathrm{s}$, as well as between the CMRs at low and high local projected densities. The CMRs typically intersect at fainter magnitudes $\left(m_{\mathrm{r}}>16\right.$ mag), but have different slopes. While a straightforward interpretation would be that the stellar populations of the $\mathrm{dE}(\mathrm{nN}) \mathrm{s}$ are both younger and less metal-rich than those of the $\mathrm{dE}(\mathrm{N}) \mathrm{s}$ (and likewise for dEs at low densities as compared to those at high densities), statistical tests and detailed comparisons with stellar population models are required 


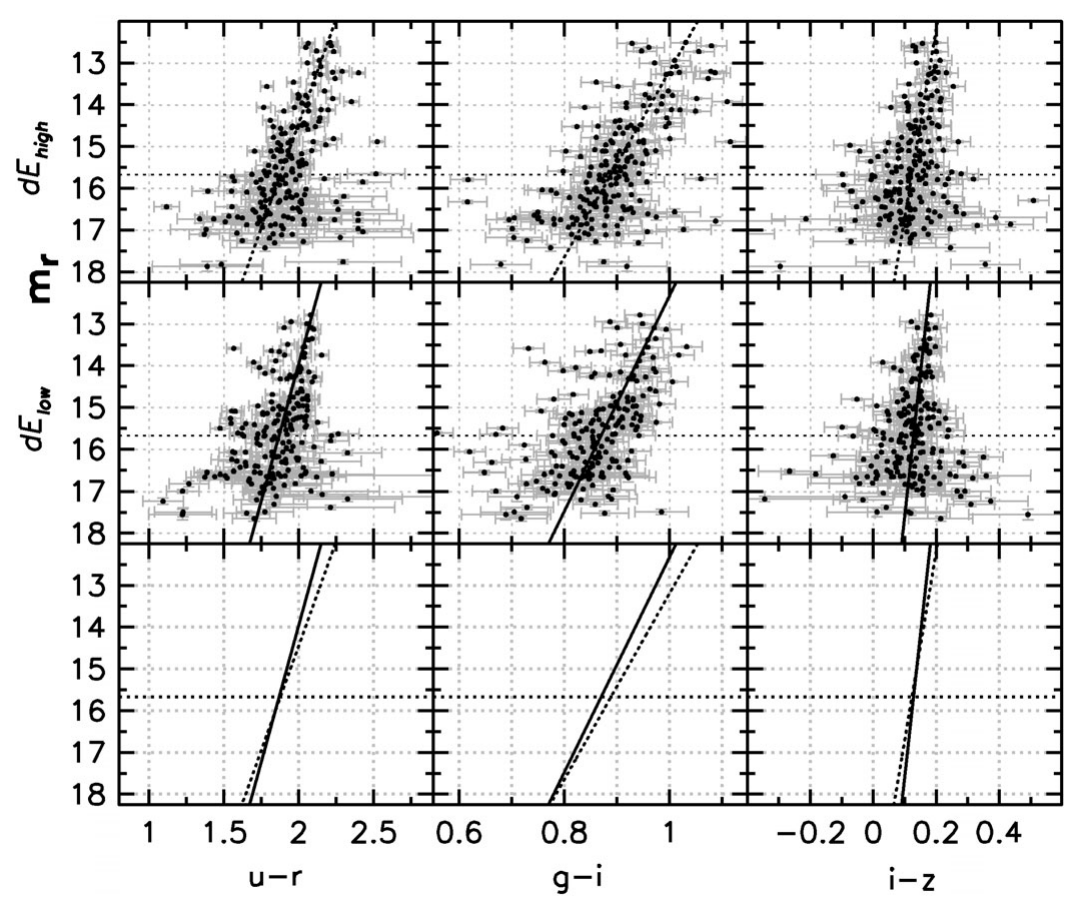

Figure 2. Colour-magnitude relations for different densities. Similar to Fig. 1, but showing the CMRs of the high-density (top row) and low-density (middle row) dE subsample, using all dEs except the $\mathrm{dE}(\mathrm{bc}) \mathrm{s}$. The corresponding linear fits are compared to each other in the bottom row.

before reliable conclusions can be drawn. These will be presented in a forthcoming paper. The existence of several dE subclasses with different shapes, clustering properties, and colour characteristics most likely indicates that dEs form through more than just a single process.

\section{Acknowledgements}

T.L. would like to thank the IAU for financial support for the participation in the Symposium. This study is based on publicly available data from the SDSS (www.sdss.org). We acknowledge support by the Swiss National Science Foundation.

\section{References}

Adelman-McCarthy, J. K., Agüeros, M. A., Allam, S. S., et al. 2007, ApJS, submitted Binggeli, B., Sandage, A., \& Tammann, G. A. 1985, AJ, 90, 1681

Gunn, J. E. \& Gott, J. R. I. 1972, ApJ, 176, 1

Jerjen, H. \& Binggeli, B., eds. 2005, Near-field cosmology with dwarf elliptical galaxies

Lisker, T., Glatt, K., Westera, P., \& Grebel, E. K. 2006b, AJ, 132, 2432

Lisker, T., Grebel, E. K., \& Binggeli, B. 2006a, AJ, 132, 497

Lisker, T., Grebel, E. K., Binggeli, B., \& Glatt, K. 2007, ApJ, in press, astro-ph/0701429

Lotz, J. M., Primack, J., \& Madau, P. 2004, AJ, 128, 163

Moore, B., Katz, N., Lake, G., Dressler, A., \& Oemler, A. 1996, Nature, 379, 613

Petrosian, V. 1976, ApJ, 209, L1

Press, W. H. 2002, Numerical recipes in C++

Schlegel, D. J., Finkbeiner, D. P., \& Davis, M. 1998, ApJ, 500, 525 


\section{Discussion}

ChILINGARIAn: With SDSS data you are supposed to be more sensitive to the presence of nuclei in dEs than with DSS data, used by Binggeli to produce VCC. Have you detected nuclei in any of the dE galaxies classified as non-nucleated in VCC?

LISKER: I have looked at the SDSS images, and would classify about a dozen objects out of $\sim 400$ as nucleated instead of non-nucleated. The SDSS is not deeper than the VCC plates, but of course still superior to photographic plates. Actually, Grant et al. (2005) suggested that there might be a continuum of nuclei strengths instead of a clear separation of $\mathrm{dE}(\mathrm{N}) \mathrm{s}$ and $\mathrm{dE}(\mathrm{nN}) \mathrm{s}$. Yet still, $\mathrm{dE}(\mathrm{N}) \mathrm{s}$ and $\mathrm{dE}(\mathrm{nN}) \mathrm{s}$ do have different properties - so this could imply a relation of nucleus strength with galaxy property.

BoEker: Can you tell us a bit more about the nature of the blue "centers"?. Are they due to ongoing star formation, and if so, isn't that unusual for spheroidal galaxies?. Could we be seeing the remnants of disks?.

LISKER: They are blue central regions and not blue nuclei (though some have several nucleus-like clumps within the center). More than one third of them show emission lines from ongoing SF; for the others, SF must have occured recently. From our simplified spectral fit to population synthesis models, we see that the old population dominates (Lisker et al. 2006, Paper II). Within the 1 Gyr after cessation of SF, one would not recognize the blue centers anymore. Their gas content is larger than that of "classical" $\mathrm{dEs}$, but lower than that of dIrrs. 4/17 objects also show disk features, and the flattening distribution implies a rather flat oblate shape. It might simply be the last stages of SF in a dE.

BEASLEY: This is a comment: $\mathrm{dE}$ nuclei in general are probably not formed purely by the disruption of their globular cluster systems since the nuclei of dEs are often redder than the GC system.

LISKER: OK. 